\title{
Hamiltonian analysis of non-relativistic non-BPS Dp-brane
}

\section{J. Klusoň}

Department of Theoretical Physics and Astrophysics, Faculty of Science, Masaryk University, Kotlářská 2, 611 37, Brno, Czech Republic

E-mail: klu@physics.muni.cz

ABSTRACT: We perform Hamiltonian analysis of non-relativistic non-BPS Dp-brane. We find the constraint structure of this theory and determine corresponding equations of motion. We further discuss property of this theory at the tachyon vacuum.

KeYwords: D-branes, Tachyon Condensation

ArXiv EPRINT: 1704.08003 


\section{Contents}

1 Introduction and summary 1

2 Non-relativistic limit of non-BPS Dp-brane 3

3 Hamiltonian formalism $\quad 6$

4 Algebra of constraints $\quad 8$

5 Equations of motion $\quad 9$

6 Non-relativistic limit of fundamental string and its Hamiltonian form 11

A Hamiltonian analysis of non-relativistic fundamental string in light-cone formulation

\section{Introduction and summary}

Holography is very useful tool for the analysis of relativistic strongly coupled field theory using Einstein classical gravity in the bulk [1-3]. One such an interesting application of the holography is the analysis of condensed matter systems, for recent review, see [4]. It turns out that non-relativistic systems play fundamental role in this analysis and hence they are now studied very intensively since they are also related to famous P. Hořava's proposal [5], for recent review, see $[6] .^{1}$

It is well known that non-relativistic symmetry can be imposed at the level of action for relativistic string or p-branes [13-19]. It is important to stress that different non-relativistic limits can be imposed, at least in principle. For example, "stringy" non-relativistic limit was firstly introduced in $[13,14]$ where the time and one spatial directions along the string are large.

There is also another non-relativistic limit of the relativistic string where only the time direction is large $[18,19]$. It turns out that this corresponds to the situation where non-relativistic string does not vibrate. In fact, it represents a collection of non-relativistic massless particles with an energy density that depends on the position of the particle along the string $[18,19]$. The action is invariant under Galilean transformations where the Poisson brackets of the generators of algebra close without central extension.

These different non-relativistic limits are very interesting and could lead to new solvable sectors of string theory, as for example in [16]. It is also interesting to study how different string theory objects behave when they world-volume actions are restricted by

\footnotetext{
${ }^{1}$ See, for example [7-12, 23].
} 
non-relativistic limiting procedure. Certainly such an interesting object is an action for non-BPS Dp-brane [25-27]. The question is whether different limiting procedures of this action do not spoil remarkable properties of the tachyon effective action. One such a nice property is that this action at its Hamiltonian formulation can describe the fate of nonBPS Dp-brane at its tachyon vacuum. For example, it was shown that at the tachyon vacuum with constant electric flux Dp-brane disappears and we are left with the gas of fundamental strings that move in the whole target space-time [29-31] which agree with the general principles of tachyon condensation in open string theory. ${ }^{2}$ The question is whether non-relativistic non-BPS Dp-brane action as was derived in [17] shares the same properties as its relativistic precursor. It is possible that limiting procedure can change property of non-BPS Dp-brane action. One such an example was studied recently in [20] where the Carrol limit of non-BPS Dp-brane was analyzed. ${ }^{3}$ We showed there that the equations of motion of Carroll non-BPS Dp-brane at its tachyon vacuum have solutions that can be interpreted as fundamental strings with however important restrictions on their momenta so that there is not exact equivalence between the conjecture that at the tachyon vacuum an unstable Dp-brane disappears and we are left with the gas of Carroll strings moving in the whole target space-time. As we will see in this paper in case of a non-relativistic non-BPS Dp-brane the situation is even worse. More explicitly, while the non-relativistic non-BPS Dp-brane action, as was derived in [17], possesses tachyon kink solution that can be interpreted as stable $\mathrm{D}(\mathrm{p}-1)$-brane [17], in case of its tachyon vacuum solution the situation is not the same. In order to show this we have to firstly determine Hamiltonian form of non-relativistic non-BPS action which is non-trivial task due to its complicated structure. We find that the bare Hamiltonian is proportional to Gauss constraint while the theory possesses $(p+1)$-primary constraints. We calculate the algebra of these constraints and show that they are the first class constraints which is a reflection of the fact that the action is invariant under world-volume diffeomorphism. Then we analyze equations of motion at the tachyon vacuum. We find, due to the structure of Hamiltonian constraint, that corresponding solutions cannot be interpreted as the solutions describing the gas of non-relativistic strings. To see this explicitly we preform also Hamiltonian analysis of nonrelativistic string in slightly different way from the original works $[18,33]$ that however coincide with them when we introduce light-cone variables as in [18].

Let us outline our results derived in this paper. We perform Hamiltonian analysis of non-relativistic non-BPS Dp-brane action, determine the structure of the constraints. We derive corresponding equations of motion and we study their behavior at the tachyon vacuum. We show that the equations of motion of the non-relativistic non-BPS Dp-brane cannot be related to the equations of motion for non-relativistic string which can be a consequence of the remarkable efficiency of the Dirac-Born-Infeld form of non-BPS Dpbrane action [28, 34-37] that is lost when we perform non-relativistic contraction.

This paper is organized as follows. In the next section (2) we review how to implement consistent non-relativistic limit for non-BPS Dp-brane. In section (3) we perform

\footnotetext{
${ }^{2}$ It was shown in [32] that in case of zero electric flux non-BPS Dp-brane at the tachyon vacuum corresponds to the gas of massless particles.

${ }^{3}$ Carroll limit of extended objects in string theory was studied previously in [21, 22, 24].
} 
Hamiltonian analysis of this theory and in section (4) we determine algebra of constraints. In section (5) we analyze corresponding equations of motion. In section (6) we review non-relativistic limit in case of fundamental string and perform its Hamiltonian analysis. Finally in appendix (A) we review Hamiltonian analysis of non-relativistic string expressed using light-cone variables.

\section{Non-relativistic limit of non-BPS Dp-brane}

Our goal is to perform well defined non-relativistic limit which is consistent in the sense that all divergences that arises during the limiting procedure, either cancel or give trivial contribution to the action. It turns out that this procedure is rather non-trivial and deserves careful treatment. To see this in more details let us consider a simple example when we start with a p-brane action in the form

$$
S=-\tilde{\tau}_{p} \int d^{p+1} \xi \sqrt{-\operatorname{det} \mathbf{A}}, \quad \mathbf{A}_{\alpha \beta}=\eta_{M N} \partial_{\alpha} \tilde{x}^{M} \partial_{\beta} \tilde{x}^{N}
$$

and try to implement non-relativistic limit, following recent paper [18]. It was argued there that we can have $p+1$ different non-relativistic limits according to the number of embedding coordinates $(0, \ldots, p)$ that are rescaled. Explicitly, we have

$$
\tilde{x}^{\mu}=\omega X^{\mu}, \quad \mu=0, \ldots, q, \quad \tilde{x}^{i}=X^{i}, \quad i=q+1, \ldots, d-1, \quad \tilde{\tau}=\frac{\tau}{\omega^{k_{q}}},
$$

where we take $\omega \rightarrow \infty$ in the end and where $k_{q}$ is integer number whose value depends on the specific limiting procedure. For example, in case of string like non-relativistic limit we have $k_{1}=0$ while in particle like limit we have $k_{0}=1$.

Note that the matrix $\mathbf{A}_{\alpha \beta}$ has the form

$$
\begin{aligned}
& \mathbf{A}_{\alpha \beta}=\omega^{2} \tilde{G}_{\alpha \beta}+\mathbf{a}_{\alpha \beta}, \\
& \tilde{G}_{\alpha \beta}=\partial_{\alpha} X^{\mu} \partial_{\beta} X_{\mu}, \quad \mathbf{a}_{\alpha \beta}=\partial_{\alpha} X^{i} \partial_{\beta} X_{i} .
\end{aligned}
$$

Now the problem is how to deal with this case since the matrix $\tilde{G}_{\alpha \beta}$ is singular for $p \neq q$. This follows from the fact that it can be written as

$$
\tilde{G}_{\alpha \beta}=E_{\alpha}^{\mu} \eta_{\mu \nu} E_{\beta}^{\nu},
$$

where $E_{\alpha}^{\mu}=\partial_{\alpha} X^{\mu}$ is $(p+1) \times(q+1)$ matrix that has rank $(q+1)$. Since $\eta_{\mu \nu}$ is $(q+1) \times(q+1)$ matrix we find that the rank of the matrix $\tilde{G}_{\alpha \beta}$ is $q+1$. On the other hand since $\tilde{G}_{\alpha \beta}$ is $(p+1) \times(p+1)$ matrix we immediately find, since $q<p$, that $\tilde{G}_{\alpha \beta}$ is singular matrix so that it is not possible to introduce its inverse. Then in order to introduce non-relativistic limit in general case we have to proceed in an systematic way as in [18] where it was shown that non-relativistic expansion is well defined for all $q$. For example, we could use explicit definition of the determinant to write resulting non-relativistic Lagrangian as was done in case of particle like limit in [18]. On the other hand there is another possibility, that was proposed previously in $[15,16]$, how to define non-relativistic limit. This procedure is based on the coupling of Dp-brane to a specific constant Ramond-Ramond background 
so that divergent contributions cancel each other. This procedure was generalized to the case of non-BPS Dp-brane in [17] and we now review its main properties. We start with Dirac-Born Infeld (DBI) part of non-BPS Dp-brane action in the flat background

$$
\begin{aligned}
S_{\mathrm{DBI}} & =-\tilde{\tau}_{p} \int d^{p+1} \xi V(\tilde{T}) \sqrt{-\operatorname{det} \mathbf{A}_{\alpha \beta}} \\
\mathbf{A}_{\alpha \beta} & =\eta_{M N} \partial_{\alpha} \tilde{x}^{M} \partial_{\beta} \tilde{x}^{N}+\tilde{F}_{\alpha \beta}+\partial_{\alpha} \tilde{T} \partial_{\beta} \tilde{T}, \quad \tilde{F}_{\alpha \beta}=\partial_{\alpha} \tilde{A}_{\beta}-\partial_{\beta} \tilde{A}_{\alpha},
\end{aligned}
$$

where $\tilde{x}^{M}, M=0, \ldots, d-1$ are embedding coordinates, $\tilde{A}_{\alpha}, \alpha=0, \ldots, p$ are world-volume gauge field, $\tilde{T}$ is the tachyon with the potential $V(\tilde{T})$ with the property that it is even function of $\tilde{T}$ that is zero for $\tilde{T}=\tilde{T}_{\text {min }}$ and $V(\tilde{T}=0)=1$. Let us now consider following non-relativistic limit $[15,17]$

$$
\begin{array}{rlrl}
\tilde{x}^{\mu} & =X^{\mu}, & \mu, \nu & =0, \ldots, p-1, \\
\tilde{x}^{a} & =\lambda X^{a}, & & \\
\tilde{\tau}_{p} & =\lambda^{-2} \tau_{p}, & a & =p, \ldots, d-1, \\
\tilde{F}_{\alpha \beta} & =\lambda F_{\alpha \beta}, \quad \tilde{A}_{\alpha}=\lambda A_{\alpha}, \quad \tilde{T}=T
\end{array}
$$

and consider the limit $\lambda \rightarrow 0 .{ }^{4}$ In other words we define relativistic limit as the limit when transverse fluctuations are small. Using (2.6) we find that the matrix $\mathbf{A}_{\alpha \beta}$ has the form

$$
\mathbf{A}_{\alpha \beta}=\mathbf{B}_{\alpha \beta}+\lambda F_{\alpha \beta}+\lambda^{2} \mathbf{C}_{\alpha \beta},
$$

where

$$
\mathbf{B}_{\alpha \beta}=\eta_{\mu \nu} \partial_{\alpha} X^{\mu} \partial_{\beta} X^{\nu}+\partial_{\alpha} T \partial_{\beta} T, \quad \mathbf{C}_{\alpha \beta}=\partial_{\alpha} X^{a} \partial_{\beta} X_{a}
$$

Then the DBI action has the form

$$
\begin{aligned}
S_{\mathrm{DBI}}= & -\frac{\tau_{p}}{\lambda^{2}} \int d^{p+1} \xi V(T) \sqrt{-\operatorname{det} \mathbf{B}} \\
& -\frac{\tau_{p}}{2} \int d^{p+1} \xi V(T) \sqrt{-\operatorname{det} \mathbf{B}}\left[\mathbf{B}^{\alpha \beta} \partial_{\beta} X^{a} \partial_{\alpha} X_{a}-\frac{1}{2} \mathbf{B}^{\alpha \beta} F_{\beta \gamma} \mathbf{B}^{\gamma \delta} F_{\delta \alpha}+O\left(\lambda^{2}\right)\right],
\end{aligned}
$$

where we defined $\mathbf{B}^{\alpha \beta}$ as inverse to $\mathbf{B}_{\alpha \beta}$

$$
\mathbf{B}_{\alpha \beta} \mathbf{B}^{\beta \gamma}=\delta_{\alpha}^{\gamma}
$$

In order to see that this is possible it is useful to introduce following notation

$$
Y^{I} \equiv\left(X^{\mu}, T\right), \quad Y^{p}=T, \quad I, J=0, \ldots, p
$$

\footnotetext{
${ }^{4}$ Note an important difference with the non-relativistic limit of stable Dp-brane in the number of the embedding fields that are not scaled. In case of non-BPS Dp-brane we have $p$ - these fields while in case of stable Dp-brane the number of these fields is $p+1$. The reason why one scalar mode is missing in case of non-BPS Dp-brane is in the specific form of the Wess-Zummino term for unstable Dp-brane which is needed for the cancelation of the divergent term that emerges when we perform the non-relativistic limit on DBI action.
} 
so that we can write $\mathbf{B}_{\alpha \beta}$ as

$$
\mathbf{B}_{\alpha \beta}=\partial_{\alpha} Y^{I} \partial_{\beta} Y_{I}=E_{\alpha}^{I} E_{\beta}^{J} \eta_{I J}, \quad I, J=0, \ldots, p, \quad \eta_{p p}=1 .
$$

Clearly $E_{\alpha}^{I}$ and $\eta_{I J}$ are $(p+1) \times(p+1)$ matrices so that $\mathbf{B}_{\alpha \beta}$ is non-singular matrix.

Using the notation introduced in (2.12) we can write the expression on the first line in $(2.9)$ as

$$
\begin{aligned}
d^{p+1} \xi \sqrt{-\operatorname{det} \mathbf{B}} & =d^{p+1} \xi \sqrt{-\operatorname{det}\left(E_{\alpha}^{I} \eta_{I J} E_{\beta}^{J}\right)} \\
& =d^{p+1} \xi \operatorname{det} E_{\alpha}^{I} \\
& =E^{0} \wedge E^{1} \wedge \cdots \wedge E^{p}=-\frac{1}{(p+1) !} \epsilon_{I_{0} \ldots I_{p}} E^{I_{0}} \wedge \ldots E^{I_{p}}
\end{aligned}
$$

where we used following normalization for Levi-Chivita symbol

$$
\epsilon^{012 \ldots p}=1, \quad \epsilon_{012 \ldots p}=-1,
$$

and where $E^{I}=E_{\alpha}^{I} d \xi^{\alpha}$. In other words the divergent term is equal to

$$
S_{\mathrm{DBI}}^{\mathrm{div}}=\frac{\tau_{p}^{2}}{\lambda^{2}(p+1) !} \int V(T) \epsilon_{I_{0} \ldots I_{p}} E^{I_{0}} \wedge \cdots \wedge E^{I_{p}} .
$$

Let us now consider Wess-Zummino term for unstable Dp-brane that expresses the coupling of this brane to the background Ramond-Ramond forms

$$
S_{\mathrm{WZ}}=\tilde{\tau}_{p} \int V(\tilde{T}) d \tilde{T} \wedge C \wedge e^{\tilde{F}} .
$$

Let us presume that there is background Ramond-Ramond $C_{p}$ form equal to

$$
C_{\mu_{0} \ldots \mu_{p-1}}=-(-1)^{p} \epsilon_{\mu_{0} \ldots \mu_{p-1}} .
$$

For this background the WZ term has the form

$$
\begin{aligned}
S_{\mathrm{WZ}} & =-\frac{\tau_{p}}{\lambda^{2}} \int V(T) \frac{1}{p !} \epsilon_{\mu_{0} \ldots \mu_{p-1}} d X^{\mu_{0}} \wedge \cdots \wedge d X^{\mu_{(p-1)}} \wedge d T \\
& =-\frac{\tau_{p}}{\lambda^{2}(p+1) !} \int V(T) \epsilon_{I_{0} \ldots I_{p}} E^{I_{0}} \wedge \cdots \wedge E^{I_{p}}
\end{aligned}
$$

and we see that it precisely cancels the divergent term (2.15). As a result we find well defined limited procedure that leads to non-relativistic non-BPS Dp-brane action in the form

$$
S_{\mathrm{NR}}=-\frac{\tau_{p}}{2} \int d^{p+1} \xi V(T) \sqrt{-\operatorname{det} \mathbf{B}}\left[\mathbf{B}^{\alpha \beta} \partial_{\beta} X^{a} \partial_{\alpha} X_{a}-\frac{1}{2} \mathbf{B}^{\alpha \beta} F_{\beta \gamma} \mathbf{B}^{\gamma \delta} F_{\delta \alpha}\right] .
$$

This action was derived previously in [17]. It was also shown there that the tachyon kink solution of the equations of motion correctly reproduces non-relativistic $\mathrm{D}(\mathrm{p}-1)$-brane action. Next step in the analysis of this action is to study its properties at the tachyon vacuum. In order to do this we have to find Hamiltonian form of this action. 


\section{Hamiltonian formalism}

The action (2.19) is rather complicated due to the fact that elements of the matrix $\mathbf{B}_{\alpha \beta}$ depend on the time derivative of $Y^{I}$. It turns out that it is useful to introduce $(p+1)$-like decomposition of the matrix $\mathbf{B}_{\alpha \beta}$ in complete analogy with $p+1$ formalism in General Relativity [38, 39] so that

$$
\begin{aligned}
\mathbf{B}^{\alpha \beta} & =\left(\begin{array}{cc}
-\frac{1}{N^{2}} & \frac{N^{j}}{N^{2}} \\
\frac{N^{i}}{N^{2}} & h^{i j}-\frac{N^{i} N^{j}}{N^{2}}
\end{array}\right), \\
\mathbf{B}_{\alpha \beta} & =\left(\begin{array}{cc}
-N^{2}+N_{i} h^{i j} N_{j} & N_{j} \\
N_{i} & h_{i j}
\end{array}\right) .
\end{aligned}
$$

If we compare $\mathbf{B}_{\alpha \beta}$ defined above with $\mathbf{B}_{\alpha \beta}=\partial_{\alpha} Y^{I} \partial_{\beta} Y_{I}$ we obtain an explicit relation between $N, N_{i}, h_{i j}$ and derivatives of $Y^{I}$

$$
\begin{gathered}
N_{i}=\partial_{i} Y^{I} \partial_{0} Y_{I}, \quad h_{i j}=\partial_{i} Y^{I} \partial_{j} Y_{I}, \\
N^{2}=\partial_{0} Y^{I}\left(\partial_{i} Y_{I} h^{i j} \partial_{j} Y_{J}-\delta_{I J}\right) \partial_{0} Y^{J} \equiv-\partial_{0} Y^{I} V_{I J} \partial_{0} Y^{J},
\end{gathered}
$$

where $h^{i j}$ is inverse to $h_{i j}$ so that $h_{i j} h^{j k}=\delta_{i}^{k}$.

If we also introduce $(p+1)$-decomposition of the gauge field we obtain an action in the form

$$
\begin{aligned}
S_{\mathrm{NR}}= & -\frac{\tau_{p}}{2} \int d^{p+1} \xi V(T) N \sqrt{h}\left[-\nabla_{n} X^{a} \nabla_{n} X_{a}+h^{i j} \partial_{i} X^{a} \partial_{j} X_{a}\right] \\
- & \frac{\tau_{p}}{4} \int d^{p+1} \xi V(T) \sqrt{h} N\left[h^{i k} h^{j l}\left(\partial_{i} A_{j}-\partial_{j} A_{i}\right)\left(\partial_{k} A_{l}-\partial_{l} A_{k}\right)\right. \\
& \left.-2 h^{i j}\left(\mathcal{L}_{n} A_{i}-a_{i} A_{\mathbf{n}}-D_{i} A_{\mathbf{n}}\right)\left(\mathcal{L}_{n} A_{j}-a_{j} A_{\mathbf{n}}-D_{j} A_{\mathbf{n}}\right)\right],
\end{aligned}
$$

where

$$
\mathcal{L}_{n} A_{i}=\frac{1}{N}\left(\partial_{0} A_{i}-N^{k} \partial_{k} A_{i}-\partial_{i} N^{k} A_{k}\right), \quad a_{i}=\frac{\partial_{i} N}{N},
$$

and where

$$
\nabla_{n} X^{a}=\frac{1}{N}\left(\partial_{0} X^{a}-N^{i} \partial_{i} X^{a}\right)
$$

Finally note that $D_{i}$ is covariant derivative compatible with the metric $h_{i j}$. Now we are ready to find conjugate momenta from the action (3.3). First of all we obtain momentum conjugate to $A_{i}$ and $A_{\mathbf{n}}$

$$
\pi^{i}=\frac{\delta L}{\delta \partial_{0} A_{i}}=\tau_{p} V(T) \sqrt{h} h^{i j}\left(\mathcal{L}_{n} A_{j}-a_{j} A_{\mathbf{n}}-D_{j} A_{\mathbf{n}}\right), \quad \pi_{\mathbf{n}} \approx 0 .
$$

Further the momentum conjugate to $X^{a}$ is equal to

$$
p_{a}=\frac{\delta L}{\delta \partial_{0} X^{a}}=\tau_{p} V(T) \sqrt{h} \nabla_{n} X_{a} .
$$

In case of $p_{I}$ we have to be more careful since $N$ and $N_{i}$ explicitly depend on $\partial_{0} Y^{I}$. Using

$$
\frac{\partial N}{\partial_{0} Y^{I}}=\frac{-V_{I J} \partial_{0} Y^{J}}{N}, \quad \frac{\partial N_{i}}{\partial_{0} Y^{I}}=\partial_{i} Y_{I}
$$


we find after some algebra

$$
\begin{aligned}
\Pi_{I} \equiv & p_{I}+\partial_{i} Y_{I} h^{i j} \partial_{j} X^{a} p_{a}+\partial_{k} Y_{I} h^{k l} \partial_{k} A_{i} \pi^{i}-\partial_{l} Y_{I} h^{l k} \partial_{i}\left[A_{k} \pi^{i}\right] \\
=-\frac{V_{I J} \partial_{0} Y^{J}}{N}\left[\frac{1}{2 \tau_{p} V \sqrt{h}} p_{a} p^{a}+\frac{\tau_{p}}{2} V \sqrt{h} h^{i j} \partial_{i} X^{a} \partial_{j} X_{a}\right. & \\
& \left.\quad+\frac{1}{2 \tau_{p} V \sqrt{h}} \pi^{i} h_{i j} \pi^{j}+\frac{\tau_{p}}{4} V \sqrt{h} h^{i j} h^{k l} F_{i j} F_{k l}+A_{\mathbf{n}} \partial_{i} \pi^{i}\right], \quad F_{i j}=\partial_{i} A_{j}-\partial_{j} A_{i} .
\end{aligned}
$$

To proceed further it is important to stress that $V_{I J}$ obey the relations

$$
\partial_{i} Y^{I} V_{I J}=0, \quad V_{I J} \eta^{J K} V_{K L}=V_{I J} V_{L}^{J}=V_{I L} .
$$

Using the first formula in (3.9) we obtain following primary constraint

$$
\mathcal{H}_{i}=\partial_{i} Y^{I} \Pi_{I}=p_{I} \partial_{i} Y^{I}+\partial_{i} X^{a} p_{a}+\partial_{i} A_{k} \pi^{k}-\partial_{k}\left[A_{i} \pi^{k}\right] \approx 0 .
$$

On the other hand if we calculate $\Pi_{I} V^{I J} \Pi_{J}$ defined in (3.9) and use (3.10) we find

$$
\begin{gathered}
\tilde{\mathcal{H}}_{T} \equiv V^{2} \Pi_{I} V^{I J} \Pi_{J}+\left(\frac{1}{2 \tau_{p} \sqrt{h}} p^{a} p_{a}+\frac{\tau_{p}}{2} V^{2} \sqrt{h} h^{i j} \partial_{i} X^{a} \partial_{j} X_{a}+\frac{1}{2 \tau_{p} \sqrt{h}} \pi^{i} h_{i j} \pi^{j}\right. \\
\left.+\frac{\tau_{p}}{4} V^{2} \sqrt{h} h^{i j} h^{k l} F_{i j} F_{k l}+V A_{\mathbf{n}} \partial_{i} \pi^{i}\right)^{2} \\
\equiv V^{2} \Pi_{I} \eta^{I J} \Pi_{J}+\mathcal{K}^{2} \approx 0 .
\end{gathered}
$$

If we compare this constraint with the constraints derived in case of non-relativistic string theory [33] we find an important difference since it was argued in [33] that the Hamiltonian constraints in non-relativistic theories should be linear in $p_{I}$ while in our case the constraint $\tilde{\mathcal{H}}_{T}$ is quadratic in $p_{I}$ and cubic in momenta $p_{a}$. In order to resolve this issue note that by definition we have that $-\Pi_{I} \eta^{I J} \Pi_{J}$ is positive. Then we can write $\tilde{\mathcal{H}}_{T}$ as

$$
\tilde{\mathcal{H}}_{T}=\mathcal{K}^{2}-\left(\sqrt{-V^{2} \Pi_{I} \eta^{I J} \Pi_{J}}\right)^{2}=\left(\mathcal{K}-V \sqrt{-\Pi_{I} \eta^{I J} \Pi_{J}}\right)\left(\mathcal{K}+V \sqrt{-\Pi_{I} \eta^{I J} \Pi_{J}}\right) \approx 0 .
$$

We see that there are two branches that can be considered as constraints. However the condition that $\mathcal{K}+V \sqrt{-\Pi_{I} V^{I J} \Pi_{J}}$ is equal to zero implies that $\mathcal{K}$ and $V \sqrt{-\Pi_{I} V^{I J} \Pi_{J}}$ should be equal to zero separately since $\mathcal{K}$ is manifestly positive definite. In other words we would have over constrained system. For that reason it is natural to consider an expression in the second bracket as a constraint and hence we propose that the Hamiltonian constraint has the form

$$
\mathcal{H}_{T}=\mathcal{K}-V \sqrt{-\Pi_{I} V^{I J} \Pi_{J}} \approx 0 .
$$

Finally we determine form of the extended Hamiltonian. Using (3.6), (3.7) and (3.9) we easily determine the bare Hamiltonian

$$
H_{B}=\int d^{p} \xi\left(p_{I} \partial_{0} Y^{I}+p_{a} \partial_{0} X^{a}+\pi^{i} \partial_{0} A_{i}-\mathcal{L}\right)=\int d^{p} \xi \pi^{i} \partial_{i} A_{\mathbf{n}}
$$

so that the extended Hamiltonian with primary constraint included has the form

$$
H_{E}=\int d^{p} \xi\left(\lambda^{0} \mathcal{H}_{T}+\lambda^{i} \mathcal{H}_{i}-\partial_{i} \pi^{i} A_{\mathbf{n}}\right)
$$


where in the following we consider $\mathcal{K}$ without the term $A_{\mathbf{n}} \partial_{i} \pi^{i}$ which is proportional to the secondary constraint $\mathcal{G}=\partial_{i} \pi^{i} \approx 0$ that arises from the requirement of the preservation of the constraint $\pi_{\mathbf{n}} \approx 0$. In the next section we determine algebra of constraints.

\section{Algebra of constraints}

Now we are ready to determine algebra of constraints. As usual we introduce smeared form of these constraints

$$
\mathbf{T}_{T}(f)=\int d^{p} \xi f \mathcal{H}_{T}, \quad \mathbf{T}_{S}\left(f^{i}\right)=\int d^{p} \xi f^{i} \mathcal{H}_{i}
$$

where $f, f^{i}$ are functions of $\xi^{\alpha}$. First of all we have

$$
\begin{array}{llrl}
\left\{\mathbf{T}_{S}\left(f^{i}\right), X^{a}\right\} & =-f^{i} \partial_{i} X^{a}, & & \left\{\mathbf{T}_{S}\left(f^{i}\right), p_{a}\right\}=-\partial_{i}\left(f^{i} p_{a}\right), \\
\left\{\mathbf{T}_{S}\left(f^{i}\right), Y^{I}\right\} & =-f^{i} \partial_{i} Y^{I}, & & \left\{\mathbf{T}_{S}\left(f^{i}\right), p_{I}\right\}=-\partial_{i}\left(f^{i} p_{I}\right), \\
\left\{\mathbf{T}_{S}\left(f^{i}\right), A_{i}\right\} & =-f^{j} \partial_{j} A_{i}+\partial_{i} f^{j} A_{j}, & & \left\{\mathbf{T}_{S}\left(f^{i}\right), \pi_{i}\right\}=-\partial_{j}\left(f^{j} \pi^{i}\right)+\partial_{k}\left(f^{i} \pi^{k}\right)
\end{array}
$$

and consequently

$$
\begin{aligned}
& \left\{\mathbf{T}_{S}\left(f^{i}\right), F_{i j}\right\}=-f^{k} \partial_{k} F_{i j}-\partial_{i} f^{k} F_{k j}-F_{i k} \partial_{j} f^{k}, \\
& \left\{\mathbf{T}_{S}\left(f^{i}\right), h_{i j}\right\}=-f^{k} \partial_{k} h_{i j}-\partial_{i} f^{k} h_{k j}-h_{i k} \partial_{j} f^{k}, \\
& \left\{\mathbf{T}_{S}\left(f^{i}\right), h^{i j}\right\}=-f^{k} \partial_{k} h^{i j}+\partial_{k} f^{i} h^{k j}+h^{i k} \partial_{k} f^{j}, \\
& \left\{\mathbf{T}_{S}\left(f^{i}\right), \sqrt{h}\right\}=-f^{i} \partial_{i} \sqrt{h}-\partial_{i} f^{i} \sqrt{h} .
\end{aligned}
$$

Using these results we easily find

$$
\begin{aligned}
\left\{\mathbf{T}_{S}\left(f^{i}\right), \mathbf{T}_{T}(g)\right\} & =\mathbf{T}_{T}\left(f^{i} \partial_{i} g\right) \\
\left\{\mathbf{T}_{S}\left(f^{i}\right), \mathbf{T}_{S}\left(g^{i}\right)\right\} & =\mathbf{T}_{S}\left(f^{k} \partial_{k} g^{j}-g^{k} \partial_{k} f^{j}\right) .
\end{aligned}
$$

Finally we proceed to the calculation of the Poisson bracket

$$
\left\{\mathbf{T}_{T}(f), \mathbf{T}_{T}(g)\right\}
$$

First of all we observe that we can write $\Pi_{I}$, using the constraint $\mathcal{H}_{i}$, as

$$
\Pi_{I}=p_{I}+\partial_{i} Y_{I} h^{i j} \mathcal{H}_{j}-\partial_{j} Y_{I} h^{j i} \partial_{i} Y^{K} p_{K} \approx V_{I}^{K} p_{K}
$$

Then we obtain very important relation

$$
\left\{\Pi_{I}(\xi), h_{i j}\left(\xi^{\prime}\right)\right\}=2 V_{I}^{J} \partial_{i} \partial_{j} Y_{J}(\xi) \delta\left(\xi-\xi^{\prime}\right)
$$

and also

$$
\left\{\Pi_{I}(\xi), h^{i j}(\xi)\right\}=-h^{i k}\left(\xi^{\prime}\right)\left\{\Pi_{I}(\xi), h_{k l}\left(\xi^{\prime}\right)\right\} h^{l j}\left(\xi^{\prime}\right)=-\left[2 h^{i k} h^{j l} V_{I}^{J} \partial_{k} \partial_{l} Y_{J}\right](\xi) \delta\left(\xi-\xi^{\prime}\right)
$$


These Poisson brackets are local which implies

$$
\left\{\int d^{p} \xi f \sqrt{-\Pi_{I} V^{I J} \Pi_{J}}, \int d^{p} \xi^{\prime} g \mathcal{K}\right\}+\left\{\int d^{p} \xi f \mathcal{K}, \int d^{p} \xi^{\prime} g \sqrt{-\Pi_{I} V^{I J} \Pi_{J}}\right\}=0 .
$$

As a result the calculation of the Poisson brackets (4.5) splits to the calculations of two Poisson brackets. The first one is equal to

$$
\left\{\int d^{p} \xi f \mathcal{K}, \int d^{p} \xi^{\prime} g \mathcal{K}\right\}=\int d^{p} \xi\left(f \partial_{i} g-g \partial_{i} f\right) h^{i j} V^{2}\left(p_{a} \partial_{j} X^{a}+F_{j k} \pi^{k}\right) .
$$

On the other hand the calculation of the second one is more involved

$$
\begin{aligned}
\left\{\int d^{p} \xi f\right. & \left.V \sqrt{-\Pi_{I} \eta^{I J} \Pi_{J}}(\xi), \int d^{p} \xi^{\prime} g V \sqrt{-\Pi_{I} \eta^{I J} \Pi_{J}}\left(\xi^{\prime}\right)\right\} \\
& =\left\{\int d^{p} \xi f V \sqrt{-p_{I} V^{I J} p_{J}}(\xi), \int d^{p} \xi^{\prime} g V \sqrt{-p_{I} V^{I J} p_{J}}\left(\xi^{\prime}\right)\right\} \\
& =\int d^{p} \xi\left(f \partial_{i} g-g \partial_{i} f\right) V^{2} h^{i j} \partial_{j} Y^{M} p_{M}
\end{aligned}
$$

where

$$
V^{I J}=\eta^{I J}-\partial_{i} Y^{I} h^{i j} \partial_{j} Y^{J}
$$

and we used the fact that

$$
\begin{aligned}
\left\{p_{I}(\xi), V^{K L}\left(\xi^{\prime}\right)\right\}= & \partial_{i}^{\prime} \delta\left(\xi-\xi^{\prime}\right) \delta_{I}^{K} h^{i j}\left(\xi^{\prime}\right) \partial_{j}^{\prime} Y^{L}\left(\xi^{\prime}\right)+\partial_{i}^{\prime} Y^{K}\left(\xi^{\prime}\right) h^{i j}\left(\xi^{\prime}\right) \partial_{j}^{\prime} \delta\left(\xi-\xi^{\prime}\right) \delta_{I}^{L} \\
& +\partial_{i}^{\prime} Y^{K} h^{i k}\left(\xi^{\prime}\right) \partial_{j}^{\prime} Y^{L}\left(\xi^{\prime}\right) h^{j l}\left(\xi^{\prime}\right)\left(\partial_{k}^{\prime} \delta\left(\xi-\xi^{\prime}\right) \partial_{l}^{\prime} Y_{I}\left(\xi^{\prime}\right)+\partial_{k}^{\prime} Y_{I}\left(\xi^{\prime}\right) \partial_{l}^{\prime} \delta\left(\xi-\xi^{\prime}\right)\right)
\end{aligned}
$$

where $\partial_{i}^{\prime} \equiv \frac{\partial}{\partial \xi_{i}^{\prime}}$. We also used the fact that $V^{I J} \partial_{i} Y_{J}=0$. Collecting (4.10) and (4.11) together we finally obtain

$$
\begin{aligned}
\left\{\mathbf{T}_{T}(f), \mathbf{T}_{T}(g)\right\} & =\int d^{p} \xi\left(f \partial_{i} g-g \partial_{i} f\right) V^{2} h^{i j}\left(p_{a} \partial_{j} X^{a}+F_{j k} \pi^{k}+p_{M} \partial_{j} Y^{M}\right) \\
& =\mathbf{T}_{S}\left(\left(f \partial_{i} g-g \partial_{i} f\right) V^{2} h^{i j}\right) .
\end{aligned}
$$

This result shows that $\mathcal{H}_{T}$ and $\tilde{\mathcal{H}}_{i}$ are first class constraints which is a consequence of the fact that non-relativistic limit does not affect world-volume structure of the theory which is still fully diffeomorphism invariant. In the next section we will analyze the equations of motion at the tachyon vacuum.

\section{Equations of motion}

Now we determine equations of motion for non-relativistic non-BPS Dp-brane in the canonical formulation. Using the extended form of the Hamiltonian $H_{E}(3.16)$ we find

$$
\begin{aligned}
\partial_{0} X^{\mu} & =\left\{X^{\mu}, H_{E}\right\}=\lambda^{0} \frac{V V^{\mu J} \Pi_{J}}{\sqrt{-\Pi_{I} V^{I J} \Pi_{J}}}+\lambda^{i} \partial_{i} X^{\mu} \\
\partial_{0} p_{\mu} & =\left\{p_{\mu}, H_{E}\right\} \\
& =\int d^{p} \xi \frac{\lambda^{0} V}{\sqrt{-\Pi_{I} V^{I J} \Pi_{J}}}\left\{p_{\mu}, \Pi_{I}(\xi)\right\} V^{I J} \Pi_{J}(\xi)+2 \partial_{i}\left[\lambda^{0} \frac{\delta \mathcal{K}}{\delta h_{i j}} \partial_{j} X_{\mu}\right]+\partial_{i}\left(\lambda^{i} p_{\mu}\right)
\end{aligned}
$$


where the Poisson bracket $\left\{p_{\mu}, \Pi_{I}\right\}$ is complicated expression whose explicit form is not important for us. At the same way we determine the equations of motion for the tachyon

$$
\begin{aligned}
\partial_{0} T= & \left\{T, H_{E}\right\}=\lambda^{0} \frac{V V^{T J} \Pi_{J}}{\sqrt{-\Pi_{I} V^{I J} \Pi_{J}}}+\lambda^{i} \partial_{i} T \\
\partial_{0} p_{T}= & \left\{p_{T}, H_{E}\right\}=\int d^{p} \xi \frac{\lambda^{0} V}{\sqrt{-\Pi_{I} V^{I J} \Pi_{J}}}\left\{p_{T}, \Pi_{I}(\xi)\right\} V^{I J} \Pi_{J}(\xi)+\frac{d V}{d T} \sqrt{-\Pi_{I} \Pi^{I}} \\
& +2 \partial_{i}\left[\lambda^{0} \frac{\delta \mathcal{K}}{\delta h_{i j}} \partial_{j} T\right]+\partial_{i}\left(\lambda^{i} p_{T}\right)-\lambda^{0} \frac{\delta \mathcal{K}}{\delta V} \frac{d V}{d T}
\end{aligned}
$$

while the equation of motion for $X^{a}, p_{a}$ have simpler form

$$
\begin{aligned}
\partial_{0} X^{a} & =\left\{X^{a}, H_{E}\right\}=\lambda^{0} \frac{p_{a}}{\tau_{p} \sqrt{h}}+\lambda^{i} \partial_{i} X^{a}, \\
\partial_{0} p_{a} & =\left\{p_{a}, H_{E}\right\}=\partial_{i}\left[\lambda^{0} V^{2} \sqrt{h} h^{i j} \partial_{j} X_{a}\right]+\partial_{i}\left(\lambda^{i} p_{a}\right) .
\end{aligned}
$$

Finally we determine equations of motion for $A_{i}, \pi^{i}$

$$
\begin{aligned}
& \partial_{0} A_{i}=\left\{A_{i}, H_{E}\right\}=\frac{\lambda^{0}}{\tau_{p} \sqrt{h}} h_{i j} \pi^{j}+\partial_{i} A_{\mathbf{n}}+\lambda^{j} F_{j i}, \\
& \partial_{0} \pi^{i}=\left\{\pi^{i}, H_{E}\right\}=\partial_{m}\left[\lambda^{0} V^{2} \tau_{p} \sqrt{h} h^{m k} h^{i l} F_{k l}\right]+\partial_{j}\left(\lambda^{i} \pi^{i}\right)-\partial_{j}\left(\lambda^{i} \pi^{j}\right) .
\end{aligned}
$$

Note that there are also constraints equations that have to be obeyed

$$
\mathcal{H}_{T}=0, \quad \mathcal{H}_{i}=0, \quad \partial_{i} \pi^{i}=0 .
$$

Now we would like to analyze these equations of motion at the tachyon vacuum $T=$ $T_{\text {min }}$, where $V\left(T_{\min }\right)=0, \frac{d V}{d T}\left(T_{\min }\right)=0, p_{T}=0, \partial_{i} T=0$. In this case the equations of motion for $X^{\mu}, p_{\mu}$ simplify considerably

$$
\partial_{0} X^{\mu}=\lambda^{i} \partial_{i} X^{\mu}, \quad \partial_{0} p_{\mu}=2 \partial_{i}\left[\lambda^{0} \frac{\delta \mathcal{K}}{\delta h_{i j}} \partial_{j} X_{\mu}\right]+\partial_{i}\left(\lambda^{i} p_{\mu}\right),
$$

while the equation of motion for $X^{a}, p_{a}, A_{i}$ and $\pi^{i}$ have the form

$$
\begin{array}{rlrl}
\partial_{0} X^{a} & =\lambda^{0} \frac{p^{a}}{\tau_{p} \sqrt{h}}+\lambda^{i} \partial_{i} X^{a}, & \partial_{0} p_{a} & =\partial_{i}\left(\lambda^{i} p_{a}\right), \\
\partial_{0} A_{i} & =\frac{\lambda^{0}}{\tau_{p} \sqrt{h}} h_{i j} \pi^{j}+\partial_{i} A_{\mathbf{n}}+\lambda^{j} F_{j i}, & \partial_{0} \pi^{i}=\partial_{j}\left(\lambda^{i} \pi^{i}\right)-\partial_{j}\left(\lambda^{i} \pi^{j}\right)
\end{array}
$$

together with the set of the constraints

$$
\begin{gathered}
\mathcal{H}_{T}\left(T_{\min }\right)=\mathcal{K}\left(T=T_{\min }\right)=\frac{p_{a} p^{a}}{2 \tau_{p} \sqrt{h}}+\frac{\pi^{i} h_{i j} \pi^{j}}{2 \tau_{p} \sqrt{h}}=0 \\
\mathcal{H}_{i}=p_{a} \partial_{i} X^{a}+p_{\mu} \partial_{i} X^{\mu}+F_{i j} \pi^{j}=0
\end{gathered}
$$

From the first equation in (5.8) we see that $\mathcal{K}$ is a sum of two positive expressions so that the only possibility to be equal to zero is to demand that $p_{a}=\pi^{i}=0$. Then the spatial 
diffeomorphism constraints imply that $\mathcal{H}_{i}=p_{\mu} \partial_{i} X^{\mu}=0$ which can be obeyed for non-zero $\partial_{i} X^{\mu}$ on condition that $p_{\mu}=0$ which is also consistent with the equation of motion for $p_{\mu}$. Finally the equations of motion for $X^{\mu}$ and $X^{a}$ have the form

$$
\partial_{0} X^{\mu}=\lambda^{i} \partial_{i} X^{\mu}, \quad \partial_{0} X^{a}=\lambda^{i} \partial_{i} X^{a}
$$

We have to demand that spatial derivatives of $X^{\mu}$ are non-zero in order to have non-singular matrix $h_{i j}$. For that reason we choose following ansatz

$$
X^{i}=\sigma^{i}, i=1, \ldots, p-1
$$

so that the equation of motion for $X^{i}$ implies $\lambda^{i}=0, i=1, \ldots, p-1$. On the other hand the equation of motion for $X^{0}$ has the form

$$
\partial_{0} X^{0}=\lambda^{p} \partial_{p} X^{0}
$$

that can be solved as $X^{0}=f(\tau+\sigma)$, where $f$ is an arbitrary function and we have imposed the condition that $\lambda^{p}=1$. Note that we cannot impose the static gauge condition $X^{0}=\tau$ since in this case we would find that $h_{i j}$ is singular. In the same way we find that $X^{a}=v^{a}(\sigma+\tau)$, where $v^{a}$ are arbitrary functions.

In summary we find that the equations of motion for non-relativistic non-BPS Dpbrane at the tachyon vacuum have solutions with rather non physical properties where the dynamics of the transverse coordinates is not related to the conjugate momenta. In other words it is not possible to identify the tachyon vacuum as the gas of the fundamental non-relativistic strings which is in sharp contrast with a similar analysis performed in case of relativistic non-BPS Dp-brane action [29-31]. In order to see this in more details let us now review non-relativistic limit for fundamental string.

\section{Non-relativistic limit of fundamental string and its Hamiltonian form}

In this section we find non-relativistic string in flat space-time, following [18]. We start with the Nambu-Goto form of string action

$$
S=-\tilde{\tau}_{F} \int d \tau d \sigma \sqrt{-\operatorname{det} G}, \quad G_{\alpha \beta}=\eta_{M N} \partial_{\alpha} \tilde{Z}^{M} \partial_{\beta} \tilde{Z}^{N}, \quad \alpha, \beta=\tau, \sigma
$$

and perform non-relativistic limit in the form

$$
\tilde{Z}^{\mu}=Z^{\mu}, \quad \mu=0,1, \quad \tilde{Z}^{i}=\lambda Z^{i}, \quad i=2, \ldots, d-1, \quad \tilde{\tau}_{F}=\lambda^{-2} \tau_{F}
$$

so that we can write

$$
G_{\alpha \beta}=\partial_{\alpha} Z^{\mu} \partial_{\beta} Z_{\mu}+\lambda^{2} \partial_{\alpha} Z^{i} \partial_{\beta} Z_{i} \equiv \mathbf{a}_{\alpha \beta}+\lambda^{2} \partial_{\alpha} Z^{i} \partial_{\beta} Z_{i}
$$

and hence

$$
S_{\mathrm{NR}}=-\frac{\tau_{F}}{\lambda^{2}} \int d \tau d \sigma \sqrt{-\operatorname{det} \mathbf{a}}-\frac{\tau_{F}}{2} \int d \tau d \sigma \sqrt{-\operatorname{det} \mathbf{a}} \mathbf{a}^{\alpha \beta} \partial_{\beta} Z^{i} \partial_{\alpha} Z_{i} .
$$


The first term in (6.4) is proportional to the integral from total derivative since

$$
-\operatorname{det} \mathbf{a}=\left[\partial_{\tau} Z^{0} \partial_{\sigma} Z^{1}-\partial_{\tau} Z^{1} \partial_{\sigma} Z^{0}\right]^{2}=\left[\partial_{\tau}\left(Z^{0} \partial_{\sigma} Z^{1}\right)-\partial_{\sigma}\left(Z^{0} \partial_{\tau} Z^{1}\right)\right]^{2}
$$

and hence

$$
\int d \tau d \sigma \sqrt{-\operatorname{det} \mathbf{a}}=\int d \tau d \sigma\left[\partial_{\tau}\left(Z^{0} \partial_{\sigma} Z^{1}\right)-\partial_{\sigma}\left(Z^{0} \partial_{\tau} Z^{1}\right)\right]
$$

so that the first term in (6.4) can be ignored. We see that in case of two world-sheet dimensions the "stringy" non-relativistic limit is well defined even with the absence of the two form background field and the resulting action has the form

$$
S_{\mathrm{NR}}=-\frac{\tau_{F}}{2} \int d \tau d \sigma \sqrt{-\operatorname{det} \mathbf{a}} \mathbf{a}^{\alpha \beta} \partial_{\beta} Z^{i} \partial_{\alpha} Z_{i}
$$

In order to find Hamiltonian form of this action we use $1+1$ notation as in section (3). Explicitly, we have

$$
\mathbf{a}_{\alpha \beta}=\left(\begin{array}{cc}
-n^{2}+n_{\sigma} h^{\sigma \sigma} n_{\sigma} & n_{\sigma} \\
n_{\sigma} & h_{\sigma \sigma}
\end{array}\right), \quad \mathbf{a}^{\alpha \beta}=\left(\begin{array}{cc}
-\frac{1}{n^{2}} & \frac{n^{\sigma}}{n^{2}} \\
\frac{n^{\sigma}}{n^{2}} & h^{\sigma \sigma}-\frac{n^{\sigma} n^{\sigma}}{n^{2}}
\end{array}\right)
$$

so that

$$
\begin{aligned}
& h_{\sigma \sigma}=\partial_{\sigma} Z^{\mu} \partial_{\sigma} Z_{\mu}, \quad h^{\sigma \sigma}=\frac{1}{\partial_{\sigma} Z^{\mu} \partial_{\sigma} Z_{\mu}}, \quad n_{\sigma}=\partial_{\tau} Z^{\mu} \partial_{\sigma} Z_{\mu} \\
& n^{2}=-\partial_{\tau} Z^{\mu}\left(\eta_{\mu \nu}-\partial_{\sigma} Z_{\mu} h^{\sigma \sigma} \partial_{\sigma} Z_{\nu}\right) \partial_{\tau} Z^{\nu} \equiv-\partial_{\tau} Z^{\mu} v_{\mu \nu} \partial_{\tau} Z^{\nu}
\end{aligned}
$$

Using this notation the action (6.7) has the form

$$
\begin{aligned}
S_{\mathrm{NR}} & =\frac{\tau_{F}}{2} \int d \tau d \sigma \sqrt{h_{\sigma \sigma}}\left(\nabla_{n} Z^{i} \nabla_{n} Z_{i}-h^{\sigma \sigma} \partial_{\sigma} Z^{i} \partial_{\sigma} Z_{i}\right), \\
\nabla_{n} Z^{i} & =\frac{1}{n}\left(\partial_{\tau} Z^{i}-n^{\sigma} \partial_{\sigma} Z^{i}\right) .
\end{aligned}
$$

Now we are ready to proceed to the Hamiltonian formalism. From (6.10) we find following conjugate momenta

$$
\begin{aligned}
k_{i} & =\tau_{F} \sqrt{h_{\sigma \sigma}} n \nabla_{n} Z_{i} \\
k_{\mu} & =\frac{\tau_{F}}{2} \frac{v_{\mu \nu} \partial_{\tau} Z^{\nu}}{n}\left[\nabla_{n} Z^{i} \nabla_{n} Z_{i}+h^{\sigma \sigma} \partial_{\sigma} Z^{i} \partial_{\sigma} Z_{i}\right]-\tau_{F} \sqrt{h_{\sigma \sigma}} \partial_{\sigma} Z_{\mu} h^{\sigma \sigma} \partial_{\sigma} Z^{i} \nabla_{n} Z_{i}
\end{aligned}
$$

that again implies

$$
\omega_{\mu} \equiv k_{\mu}+\partial_{\sigma} Z_{\mu} h^{\sigma \sigma} \partial_{\sigma} Z^{i} k_{i}=\frac{v_{\mu \nu} \partial_{\tau} Z^{\nu}}{n}\left[\frac{k_{i} k^{i}}{2 \tau_{F} \sqrt{h_{\sigma \sigma}}}+\frac{\tau_{F}}{2} \sqrt{h_{\sigma \sigma}} h^{\sigma \sigma} \partial_{\sigma} Z^{i} \partial_{\sigma} Z_{i}\right]
$$

Since $v_{\mu \nu}$ obey the relations

$$
v_{\mu \nu} \partial_{\sigma} Z^{\nu}=0, \quad v_{\mu \nu} \eta^{\nu \rho} v_{\rho \sigma}=v_{\mu \nu}
$$


we obtain two primary constraints

$$
\begin{aligned}
& \mathcal{H}_{\sigma}=\omega_{\mu} \partial_{\sigma} Z^{\mu}=k_{\mu} \partial_{\sigma} Z^{\mu}+k_{i} \partial_{\sigma} Z^{i} \approx 0, \\
& \tilde{\mathcal{H}}_{\tau}=\omega_{\mu} v^{\mu \nu} \omega_{\nu}+\left[\frac{k_{i} k^{i}}{2 \tau_{F} \sqrt{h_{\sigma \sigma}}}+\frac{\tau_{F}}{2} \sqrt{h_{\sigma \sigma}} h^{\sigma \sigma} \partial_{\sigma} Z^{i} \partial_{\sigma} Z_{i}\right]^{2} \equiv \Sigma^{2}+\omega_{\mu} v^{\mu \nu} \omega_{\nu} \approx 0 .
\end{aligned}
$$

Since $-\omega_{\mu} v^{\mu \nu} \omega_{\nu}$ is positive we can write $\tilde{\mathcal{H}}_{\tau}$ as

$$
\tilde{\mathcal{H}}_{\tau}=\left(\Sigma-\sqrt{-\omega_{\mu} v^{\mu \nu} \omega_{\nu}}\right)\left(\Sigma+\sqrt{-\omega_{\mu} v^{\mu \nu} \omega_{\nu}}\right) \approx 0 .
$$

Using the same arguments as in section (3) we can argue that the Hamiltonian constraint $\mathcal{H}_{\tau}$ has the form

$$
\mathcal{H}_{\tau}=\Sigma-\sqrt{-\omega_{\mu} v^{\mu \nu} \omega_{\nu}} \approx 0
$$

and we can again show that it is the first class constraints together with $\mathcal{H}_{\sigma}$. Further, it is also easy to see, using (6.12), that the bare Hamiltonian is equal to zero so that the extended Hamiltonian is a sum of two class constraints

$$
H_{E}=\int d \sigma\left(\lambda^{\tau} \mathcal{H}_{\tau}+\lambda^{\sigma} \mathcal{H}_{\sigma}\right) .
$$

The equations of motion for $Z^{\mu}, k_{\mu}$ that follow from (6.17) have the form

$$
\begin{aligned}
\partial_{\tau} Z^{\mu}=\left\{Z^{\mu}, H_{E}\right\}= & \lambda^{\tau} \frac{v^{\mu \nu} \omega_{\nu}}{\sqrt{-\omega_{\mu} v^{\mu \nu} \omega_{\nu}}}+\lambda^{\sigma} \partial_{\sigma} Z^{\mu} \\
\partial_{\tau} k_{\mu}=\left\{k_{\mu}, H_{E}\right\}= & -\partial_{\sigma}\left[\lambda^{\tau} \frac{h^{\sigma \sigma} k_{\mu} \partial_{\sigma} Z^{\nu} k_{\nu}}{\sqrt{-\omega_{\mu} v^{\mu \nu} \omega_{\nu}}}\right]-\partial_{\sigma}\left[\lambda^{\tau} \frac{\partial_{\sigma} Z^{\rho} k_{\rho} \partial_{\sigma} Z^{\sigma} k_{\sigma} \partial_{\sigma} Z_{\mu}}{h_{\sigma \sigma}^{2} \sqrt{-\omega_{\mu} v^{\mu \nu} \omega_{\nu}}}\right] \\
& -\partial_{\sigma}\left[\lambda^{\tau} \frac{1}{2 h_{\sigma \sigma}} \partial_{\sigma} Z_{\mu} \Sigma\right]+\partial_{\sigma}\left(\lambda^{\sigma} k_{\mu}\right) .
\end{aligned}
$$

Finally the equations of motion for $Z^{i}, p_{i}$ have the form

$$
\begin{aligned}
\partial_{\tau} Z^{i} & =\left\{Z^{i}, H_{E}\right\}=\lambda^{\tau} \frac{k^{i}}{\tau_{F} \sqrt{h_{\sigma \sigma}}}+\lambda^{\sigma} \partial_{\sigma} Z^{i}, \\
\partial_{\tau} k_{i}=\left\{k_{a}, H_{E}\right\} & =\partial_{\sigma}\left[\lambda^{\tau} \frac{\tau_{F}}{\sqrt{h_{\sigma \sigma}}} \partial_{\sigma} Z_{i}\right]+\partial_{\sigma}\left(\lambda^{\sigma} k_{i}\right) .
\end{aligned}
$$

Note also that the system has to obey the constraints $\mathcal{H}_{\tau}=0, \mathcal{H}_{\sigma}=0$. Let us try to solve these equations of motion at the static gauge where

$$
Z^{1}=\sigma, \quad Z^{0}=\tau
$$

In this gauge we have $h_{\sigma \sigma}=1, v^{\tau \tau}=-1, v^{\tau \sigma}=0, v^{\sigma \sigma}=0$. Then the equation of motion for $Z^{1}$ implies $\lambda^{\sigma}=0$ while the equation of motion for $Z^{0}$ implies

$$
\lambda^{\tau}=-1 \text {. }
$$

On the other hand $k_{1}$ can be determined using the spatial diffeomorphism constraint $\mathcal{H}_{\sigma}=0$ while $k_{0}$ can be determined using $\mathcal{H}_{\tau}=0$

$$
k_{1}=-k_{i} \partial_{\sigma} Z^{i}, \quad k_{0}=\Sigma .
$$


Using these results in (6.19) we obtain that they simplify considerably

$$
\partial_{\tau} Z^{i}=\frac{k^{i}}{\tau_{F}}, \quad \partial_{\tau} K_{i}=\tau_{F} \partial_{\sigma}^{2} Z_{i}
$$

that in the end lead to the wave equation

$$
\partial_{\tau}^{2} Z^{i}-\partial_{\sigma}^{2} Z^{i}=0
$$

that correspond to the string vibrations in the transverse space with agreement with [33].

Finally we compare the Hamiltonian of the non-relativistic string derived here with the Hamiltonian found recently in [18]. In order to do this we introduce light-cone coordinates

$$
\begin{aligned}
r & =Z^{0}-Z^{1}, & s & =Z^{0}+Z^{1}, \\
p_{r} & =\frac{1}{2}\left(p_{0}-p_{1}\right), & p_{s} & =\frac{1}{2}\left(p_{0}+p_{1}\right),
\end{aligned}
$$

so that $\left\{r, p_{r}\right\}=1,\left\{r, p_{s}\right\}=0,\left\{s, p_{s}\right\}=1,\left\{s, p_{r}\right\}=0$. In terms of the variables (6.25) we find explicit form of $v^{\mu \nu}$ and $h_{\sigma \sigma}$

$$
\begin{aligned}
h_{\sigma \sigma} & =-r^{\prime} s^{\prime}, & r^{\prime} & \equiv \partial_{\sigma} r, \\
v^{\tau \tau} & =-1+\frac{1}{4 r^{\prime} s^{\prime}}\left(r^{\prime 2}+2 r^{\prime} s^{\prime}+s^{\prime 2}\right), & v^{\tau \sigma} & =\frac{1}{4 r^{\prime} s^{\prime}}\left(s^{\prime 2}-r^{\prime 2}\right), \\
v^{\sigma \tau} & =\frac{1}{4 r^{\prime} s^{\prime}}\left(s^{\prime 2}-r^{\prime 2}\right), & v^{\sigma \sigma} & =1+\frac{1}{4 r^{\prime} s^{\prime}}\left(r^{\prime 2}-2 r^{\prime} s^{\prime}+s^{\prime 2}\right) .
\end{aligned}
$$

Using these formulas we easily determine Hamiltonian constraint in the light cone variables to be equal to

$$
\mathcal{H}_{\tau}=\frac{1}{\sqrt{-r^{\prime} s^{\prime}}}\left[p_{i} p^{i}+\tau_{F}^{2} Z_{i}^{\prime} Z_{i}^{\prime}\right]-\frac{1}{\sqrt{-r^{\prime} s^{\prime}}}\left(p_{r} r^{\prime}-p_{s} s^{\prime}\right) \approx 0
$$

that agrees with the Hamiltonian constraint found in [18]. For reader's convenience we repeat the Hamiltonian analysis performed in [18] in appendix.

\section{A Hamiltonian analysis of non-relativistic fundamental string in light- cone formulation}

In this appendix we review the Hamiltonian analysis of non-relativistic string in the light cone variables. We define these variables as in previous section

$$
Z^{0}=\frac{1}{2}(r+s), \quad Z^{1}=\frac{1}{2}(s-r)
$$

so that we easily find that matrix $\mathbf{a}_{\alpha \beta}$ and its inverse $\mathbf{a}^{\alpha \beta}$ have the form

$$
\begin{array}{rlrl}
\mathbf{a}_{\tau \tau} & =-\dot{r} \dot{s}, & \mathbf{a}_{\tau \sigma}=-\frac{1}{2}\left(\dot{r} s^{\prime}+\dot{s} r^{\prime}\right), & \mathbf{a}_{\sigma \sigma}=-r^{\prime} s^{\prime}, \\
\mathbf{a}^{\tau \tau}=\frac{4 r^{\prime} s^{\prime}}{\left(\dot{r} s^{\prime}-\dot{s} r^{\prime}\right)^{2}}, & \mathbf{a}^{\tau \sigma}=-2 \frac{\left(\dot{r} s^{\prime}+\dot{s} r^{\prime}\right)}{\left(\dot{r} s^{\prime}-\dot{s} r^{\prime}\right)^{2}}, & \mathbf{a}^{\sigma \sigma}=\frac{4 \dot{r} \dot{s}}{\left(\dot{r} s^{\prime}-\dot{s} r^{\prime}\right)^{2}}
\end{array}
$$


so that non-relativistic string action has the form

$$
S_{\mathrm{NR}}^{\text {l.c. }}=\tau_{F} \int d \tau d \sigma \frac{1}{\dot{r} s^{\prime}-\dot{s} r^{\prime}}\left(-r^{\prime} s^{\prime} \dot{Z}^{i} \dot{Z}_{i}+\left(\dot{r} s^{\prime}+\dot{s} r^{\prime}\right) \dot{Z}^{i} Z_{i}^{\prime}-\dot{r} \dot{s} Z^{\prime i} Z_{i}^{\prime}\right) \equiv \int d \tau d \sigma \mathcal{L}_{\mathrm{NR}} .
$$

From (A.3) we find conjugate momenta

$$
\begin{aligned}
& p_{r}=\frac{\partial \mathcal{L}_{\mathrm{NR}}}{\partial \dot{r}}=-\frac{s^{\prime}}{\left(\dot{r} s^{\prime}-\dot{s} r^{\prime}\right)} \mathcal{L}_{\mathrm{NR}}+\frac{\tau_{F}}{\dot{r} s^{\prime}-\dot{s} r^{\prime}}\left(s^{\prime} \dot{Z}^{i} Z_{i}^{\prime}-\dot{s} Z^{\prime i} Z_{i}^{\prime}\right), \\
& p_{s}=\frac{\partial \mathcal{L}_{\mathrm{NR}}}{\partial \dot{s}}=\frac{r^{\prime}}{\left(\dot{r} s^{\prime}-\dot{s} r^{\prime}\right)} \mathcal{L}_{\mathrm{NR}}+\frac{\tau_{F}}{\dot{r} s^{\prime}-\dot{s} r^{\prime}}\left(r^{\prime} \dot{Z}^{i} Z_{i}^{\prime}-\dot{r} Z^{\prime i} Z_{i}^{\prime}\right), \\
& p_{i}=\frac{\partial \mathcal{L}_{\mathrm{NR}}}{\partial \dot{Z}^{i}}=\frac{\tau_{F}}{\dot{r} s^{\prime}-\dot{s} r^{\prime}}\left(-2 r^{\prime} s^{\prime} \dot{Z}_{i}+\left(\dot{r} s^{\prime}+\dot{s} r^{\prime}\right) Z_{i}^{\prime}\right)
\end{aligned}
$$

so that we easily find that the bare Hamiltonian vanish

$$
H_{B}=\int d \sigma\left(p_{r} \dot{r}+p_{s} \dot{s}+p_{i} \dot{Z}^{i}-\mathcal{L}_{\mathrm{NR}}\right)=0 .
$$

On the other hand using (A.4) we find following primary constraints

$$
\mathcal{H}_{\sigma}=p_{r} r^{\prime}+p_{s} s^{\prime}+p_{i} Z^{\prime i} \approx 0
$$

and also

$$
\mathcal{H}_{\tau}^{\text {l.c. }} \equiv-p_{r} r^{\prime}+p_{s} s^{\prime}+\frac{1}{2 \tau_{F}}\left(p_{i} p^{i}+\tau_{F}^{2} Z_{i}^{\prime} Z^{\prime i}\right) \approx 0
$$

that were previously derived in [18].

\section{Acknowledgments}

This work was supported by the Grant Agency of the Czech Republic under the grant P201/12/G028.

Open Access. This article is distributed under the terms of the Creative Commons Attribution License (CC-BY 4.0), which permits any use, distribution and reproduction in any medium, provided the original author(s) and source are credited.

\section{References}

[1] J.M. Maldacena, The Large- $N$ limit of superconformal field theories and supergravity, Int. J. Theor. Phys. 38 (1999) 1113 [Adv. Theor. Math. Phys. 2 (1998) 231] [hep-th/9711200] [INSPIRE].

[2] S.S. Gubser, I.R. Klebanov and A.M. Polyakov, Gauge theory correlators from noncritical string theory, Phys. Lett. B 428 (1998) 105 [hep-th/9802109] [INSPIRE].

[3] E. Witten, Anti-de Sitter space and holography, Adv. Theor. Math. Phys. 2 (1998) 253 [hep-th/9802150] [INSPIRE]. 
[4] S.A. Hartnoll, A. Lucas and S. Sachdev, Holographic quantum matter, arXiv:1612.07324 [INSPIRE].

[5] P. Hořava, Quantum Gravity at a Lifshitz Point, Phys. Rev. D 79 (2009) 084008 [arXiv: 0901.3775] [INSPIRE].

[6] A. Wang, Hoŕava gravity at a Lifshitz point: a progress report, Int. J. Mod. Phys. D 26 (2017) 1730014 [arXiv:1701.06087] [inSPIRE].

[7] E. Bergshoeff et al., Carroll versus Galilei Gravity, JHEP 03 (2017) 165 [arXiv: 1701.06156] [INSPIRE].

[8] E.A. Bergshoeff and J. Rosseel, Three-dimensional extended Bargmann supergravity, Phys. Rev. Lett. 116 (2016) 251601 [arXiv: 1604.08042] [INSPIRE].

[9] J. Hartong, Gauging the Carroll algebra and ultra-relativistic gravity, JHEP 08 (2015) 069 [arXiv: 1505.05011] [INSPIRE].

[10] E. Bergshoeff, J. Rosseel and T. Zojer, Newton-Cartan (super)gravity as a non-relativistic limit, Class. Quant. Grav. 32 (2015) 205003 [arXiv: 1505. 02095] [INSPIRE].

[11] R. Andringa, E. Bergshoeff, S. Panda and M. de Roo, Newtonian gravity and the Bargmann algebra, Class. Quant. Grav. 28 (2011) 105011 [arXiv:1011.1145] [INSPIRE].

[12] E. Bergshoeff, J. Rosseel and T. Zojer, Newton-Cartan supergravity with torsion and Schrödinger supergravity, JHEP 11 (2015) 180 [arXiv:1509.04527] [INSPIRE].

[13] J. Gomis and H. Ooguri, Nonrelativistic closed string theory, J. Math. Phys. 42 (2001) 3127 [hep-th/0009181] [INSPIRE].

[14] U.H. Danielsson, A. Guijosa and M. Kruczenski, IIA/B, wound and wrapped, JHEP 10 (2000) 020 [hep-th/0009182] [INSPIRE].

[15] J. Gomis, K. Kamimura and P.K. Townsend, Non-relativistic superbranes, JHEP 11 (2004) 051 [hep-th/0409219] [INSPIRE].

[16] J. Gomis, J. Gomis and K. Kamimura, Non-relativistic superstrings: A New soluble sector of $A d S_{5} \times S^{5}, J H E P 12(2005) 024$ [hep-th/0507036] [INSPIRE].

[17] J. Kluson, Non-relativistic non-BPS Dp-brane, Nucl. Phys. B 765 (2007) 185 [hep-th/0610073] [INSPIRE].

[18] C. Batlle, J. Gomis and D. Not, Extended Galilean symmetries of non-relativistic strings, JHEP 02 (2017) 049 [arXiv: 1611.00026] [INSPIRE].

[19] J. Gomis and P.K. Townsend, The Galilean superstring, JHEP 02 (2017) 105 [arXiv: 1612.02759] [INSPIRE].

[20] J. Kluson, Carroll limit of non-BPS Dp-brane, JHEP 05 (2017) 108 [arXiv:1702.08685] [INSPIRE].

[21] E. Bergshoeff, J. Gomis and G. Longhi, Dynamics of Carroll particles, Class. Quant. Grav. 31 (2014) 205009 [arXiv:1405.2264] [INSPIRE].

[22] E. Bergshoeff, J. Gomis and L. Parra, The symmetries of the Carroll superparticle, J. Phys. A 49 (2016) 185402 [arXiv: 1503.06083] [INSPIRE].

[23] E.A. Bergshoeff, J. Hartong and J. Rosseel, Torsional Newton-Cartan geometry and the Schrödinger algebra, Class. Quant. Grav. 32 (2015) 135017 [arXiv: 1409.5555] [INSPIRE]. 
[24] B. Cardona, J. Gomis and J.M. Pons, Dynamics of Carroll strings, JHEP 07 (2016) 050 [arXiv: 1605. 05483] [INSPIRE].

[25] A. Sen, Supersymmetric world volume action for non-BPS D-branes, JHEP 10 (1999) 008 [hep-th/9909062] [INSPIRE].

[26] E.A. Bergshoeff, M. de Roo, T.C. de Wit, E. Eyras and S. Panda, T duality and actions for non-BPS D-branes, JHEP 05 (2000) 009 [hep-th/0003221] [INSPIRE].

[27] J. Kluson, Proposal for non-BPS D-brane action, Phys. Rev. D 62 (2000) 126003 [hep-th/0004106] [INSPIRE].

[28] A. Sen, Tachyon dynamics in open string theory, Int. J. Mod. Phys. A 20 (2005) 5513 [hep-th/0410103] [INSPIRE].

[29] A. Sen, Fundamental strings in open string theory at the tachyonic vacuum, J. Math. Phys. 42 (2001) 2844 [hep-th/0010240] [INSPIRE].

[30] A. Sen, Open and closed strings from unstable D-branes, Phys. Rev. D 68 (2003) 106003 [hep-th/0305011] [INSPIRE].

[31] J. Kluson, Note about unstable D-branes with dynamical tension, Phys. Rev. D 94 (2016) 046004 [arXiv: 1605.09510] [INSPIRE].

[32] J. Kluson, Remark about non-BPS Dp-brane at the tachyon vacuum moving in curved background, Phys. Rev. D 72 (2005) 106005 [hep-th/0504062] [INSPIRE].

[33] J. Gomis and F. Passerini, Rotating solutions of non-relativistic string theory, Phys. Lett. B 617 (2005) 182 [hep-th/0411195] [INSPIRE].

[34] D. Erkal, D. Kutasov and O. Lunin, Brane-antibrane dynamics from the tachyon DBI action, arXiv:0901.4368 [INSPIRE].

[35] D. Kutasov, A geometric interpretation of the open string tachyon, hep-th/0408073 [INSPIRE].

[36] D. Kutasov and V. Niarchos, Tachyon effective actions in open string theory, Nucl. Phys. B 666 (2003) 56 [hep-th/0304045] [INSPIRE].

[37] A. Sen, Geometric tachyon to universal open string tachyon, JHEP 05 (2007) 035 [hep-th/0703157] [INSPIRE].

[38] E. Gourgoulhon, 3+1 formalism and bases of numerical relativity, gr-qc/0703035 [INSPIRE].

[39] R.L. Arnowitt, S. Deser and C.W. Misner, The dynamics of general relativity, Gen. Rel. Grav. 40 (2008) 1997 [gr-qc/0405109] [INSPIRE]. 